Original Article

\title{
Probing the effects of oxygen-related defects on the optical and luminescence properties in $\mathrm{CaCu}_{3} \mathrm{Ti}_{4} \mathrm{O}_{12}$ ceramics
}

\author{
A.A. Felix ${ }^{\mathrm{a}, *}$, M. Spreitzer ${ }^{\mathrm{b}}$, D. Vengust ${ }^{\mathrm{b}}$, D. Suvorov ${ }^{\mathrm{b}}$, M.O. Orlandi ${ }^{\mathrm{a}}$ \\ a Physical Chemistry Department, Chemistry Institute, São Paulo State University (UNESP), Araraquara, São Paulo, Brazil \\ b Advanced Materials Department, Jozef Stefan Institute, Ljubljana, Slovenia
}

\section{A R T I C L E I N F O}

\section{Keywords:}

$\mathrm{CaCu}_{3} \mathrm{Ti}_{4} \mathrm{O}_{12}$

Oxygen

Defects

Electronic structure

Spectroscopic techniques

\begin{abstract}
A B S T R A C T
The formation of oxygen-related lattice defects and their influence on optical and electronic properties in $\mathrm{CaCu}_{3} \mathrm{Ti}_{4} \mathrm{O}_{12}$ (CСTO) ceramics were studied by controlling the oxygen partial pressure during the sintering process at high temperatures. The samples were analyzed using complementary bulk spectroscopic techniques such as photoluminescence spectroscopy, ultraviolet-visible spectroscopy, Fourier-transform infrared spectroscopy, and electron paramagnetic resonance. Our comprehensive study shows that the oxygen content during sintering process exerts considerable control over the type and concentration of oxygen-related defects in the crystalline ССТO structure. Our results also suggest that the formation of different types of oxygen-related defects in the structure modifies electronic energy levels and thus directly affects the electronic and optical properties of the ССТO phase. These results provided further insight into the important role of oxygen in the crystalline defect arrangement in polycrystalline CCTO ceramics.
\end{abstract}

\section{Introduction}

The perovskite-type calcium copper titanate $\mathrm{CaCu}_{3} \mathrm{Ti}_{4} \mathrm{O}_{12}$ (CCTO) has been widely investigated due to its giant dielectric permittivity (up to $10^{6}$ ) at a wide range of temperatures and frequencies, which occurs without phase transition [1,2]. The colossal dielectric response was initially attributed to the intrinsic spatial structure of the CCTO phase, but further studies indicated only minor contributions from these features to the dielectric response of the material [3,4]. Consequently, phenomenological models based on extrinsic mechanisms have been proposed to explain this unique property of ССТО [5,6,7].

The issue of the dielectric response of CCTO ceramics notwithstanding, the mechanisms responsible for controlling other properties in this material (such as hopping conduction, gas sensing, and optical properties) are still not fully understood $[8,9,10]$. Normally, these properties in perovskite oxides are found to be controlled by oxygenrelated and/or cation-oxygen complex defects [8,11]. The formation of point defects, such as oxygen or cation vacancies, has also been reported to play an important role in the electrical and optical properties of the CCTO phase. Copper and titanium reduction, as well as copper oxidation, have been reported as the main mechanisms controlling the semiconducting nature of these ceramics $[10,12,13]$. The formation of $\mathrm{TiO}_{5} . \mathrm{V}_{\mathrm{o}}^{\cdot}-\mathrm{Cu}_{\mathrm{Cu}}^{\prime}$ and antisite defects has also been found to influence the electrical behavior of CCTO $[7,14,15]$. In contrast, the optical behavior of this perovskite has been attributed to the presence of cation-oxygen clusters activated by oxygen-related defects, such as $\left[\mathrm{TiO}_{5} . \mathrm{V}_{o}^{*}\right]$ and $\left[\mathrm{CaO}_{11} . \mathrm{V}_{o}^{*}\right][8,16]$. The point defect formation issue in the СCTO phase is therefore not fully understood and, in some of the aforementioned cases, requires clearer experimental evidence based on bulk characterization techniques.

Our previous study has shown that the oxygen partial pressure during the sintering process is an important parameter controlling the phase stability, stoichiometry, and decomposition process of the CCTO phase, as well as densification and grain growth mechanisms in polycrystalline ceramics [17]. The current study used complementary bulk spectroscopic techniques in an attempt to obtain a deeper understanding of the role of oxygen in the formation of oxygen-related defects in the crystalline lattice of ССТО ceramics and the correlation between these defects and electronic and photoluminescence properties. Our results show that oxygen exerts considerable control over the type and the concentration of defects in the crystalline structure and plays an important role in the modulation of electronic and optical properties in the CCTO phase.

\footnotetext{
* Corresponding author.

E-mail address: aafelixy@yahoo.com.br (A.A. Felix).
} 


\section{Experimental procedure}

\subsection{Synthesis procedure}

Bulk polycrystalline CCTO samples were prepared using a solidstate reaction method. The pellets were sintered at $1100{ }^{\circ} \mathrm{C}$ for $3 \mathrm{~h}$ at different oxygen partial pressures. This work is a follow-up study on the same samples investigated previously. More details about the synthesis procedure can be found in our previous articles $[17,18]$. The samples and their respective oxygen partial pressure conditions during the sintering process that were studied herein were: ССTO10 $\left(10.0 \% \mathrm{pO}_{2}\right)$, CCTO21 $\left(21.0 \% \mathrm{pO}_{2}\right)$, CCTO50 $\left(50.0 \% \mathrm{pO}_{2}\right)$, CCTO75 $\left(75.0 \% \mathrm{pO}_{2}\right)$, and CCTO100 $\left(100.0 \% \mathrm{pO}_{2}\right)$. The samples were chosen based on our previous results, which were based on X-ray powder diffraction (XRPD) and scanning electron microscopy (SEM), and which showed that these systems present phase purity higher than $99 \mathrm{wt} \%$ [17]. Thus, the spectroscopic results extracted from these experiments can be attributed to the characteristics of CCTO phase.

\subsection{Characterizations}

Photoluminescence (PL) spectra were collected using a monochromator (Monospec 27, Thermal Jarrel Ash) coupled to a photomultiplier (R446, Hamamatsu Photonics). A krypton ion laser ( $\lambda=350 \mathrm{~nm}$; Innova $90 \mathrm{~K}$, Coherent) was used as the excitation source, and maximum laser power was kept constant at $40 \mathrm{~mW}$. Fouriertransform infrared (FTIR) analyses (Vertex 70, Bruker) were performed in attenuated total reflection (ATR) mode with 64 scans and at a resolution of $4 \mathrm{~cm}^{-1}$. Ultraviolet-visible (UV-vis) spectroscopy data (Lambda $1050 \mathrm{UV} / \mathrm{Vis}$, Perkin Elmer) were recorded in reflectance mode. The PL, FTIR, and UV-VIS spectroscopic analyses were performed at room temperature on the as-prepared pellets. In order to avoid secondary effects, no additional experimental procedures, such as post-annealing treatment or surface polishing, were performed on the pellets as part of these analyses. Electron paramagnetic resonance (EPR) spectroscopy (MS300, Magnettech) was performed at an X-band (about $9.3 \mathrm{GHz}$ ) using portions of the as-prepared pellets (mass of approximately $5 \mathrm{mg}$ ). The g-factor was calibrated using 2,2-diphenyl-1-picrylhydrazine (DPPH).

\section{Results and discussion}

PL measurements have been successfully used to provide information on degrees of short- and medium-range order-disorder and have been found to be correlated with electronic and structural properties of oxides $[19,20]$. Fig. 1a shows the PL spectra of CCTO samples sintered at different oxygen partial pressures. All of the PL spectra exhibited a wide emission band in the visible optical range. This type of emission band is typically associated with multiphonon and multilevel processes [19]. It is composed of sub-bands, which can represent different types of electronic transitions due to the existence of energy levels in the band gap of the material that are commonly associated with specific structural defects [21]. The decreases in overall PL intensity without shifts in peak positions combined with the increase in oxygen partial pressure indicate an increase in short- and medium-range lattice ordering. In addition, the decrease in PL emission at long wavelengths may suggest a decrease in the concentration of specific defects in CCTO material [21].

The PL spectra were deconvoluted using a Gaussian line shape in Fig. 1c-d and S1 (see Supplementary Material) to identify the sub-bands of the overall emission. The PL spectra of all of the samples were best fit after deconvolution into three PL sub-bands with maximum peak center intensities at approximately 445 ( \pm 5$) \mathrm{nm}$ (blue emission), 515 ( \pm 5 ) $\mathrm{nm}$ (green emission), and $575( \pm 5) \mathrm{nm}$ (yellow emission). The emission intensity areas (\%) of the sub-bands confirm that the increase in oxygen partial pressure leads to a shift of the major PL emission contribution from the yellow emission to the blue emission, as seen in Fig. 1b. The emission energies estimated at $2.80 \mathrm{eV}$ (blue emission), $2.45 \mathrm{eV}$ (green emission), and $2.20 \mathrm{eV}$ (yellow emission) have mainly been assigned to the oxygen vacancies $\left(V_{o}^{x}, V_{o}^{\cdot}, V_{o}^{\cdot *}\right)$ in oxide materials [8,22,23].

Recently, Xiao et al. proposed a theoretical model indicating that the band gap energy of CCTO may be modulated by oxygen-related defects [24]. The optical band gap energies for the CCTO samples sintered at different oxygen partial pressures (Fig. 2) were estimated using the method proposed by Kubelka-Munk, which assumes a direct allowed transition $(n=0.5)[20,25]$. The results are consistent with the band gap value of approximately $3.0 \mathrm{eV}$ reported for the CCTO phase [8]. The estimated optical band gap value also increased as oxygen partial pressure increased, as shown in the right inset in Fig. 2. It is known that the radiative transition to the upper conduction band can be seriously quenched (and thus reduce band gap values) when the formation of point defects increases the probability of a non-radiative recombination [26]. Moreover, the electronic band structure of CCTO is thought to consist of an upper valence band of O2p - Cu3d states and a conduction band of O2p - Ti3d states, with the existence of deep-level trap states associated with the formation of different types of oxygen vacancies $\left(V_{o}^{x}, V_{o}^{\cdot}, V_{o}^{*}\right)[24]$.

EPR has been an effective technique when probing oxygen-related defects in oxide materials [11,27]. Fig. 3 shows the EPR lines at room temperature for the samples sintered at different oxygen partial pressures. A single Lorentzian line shape (see Figure S2) was observed in all of the samples, with a symmetric signal centered around $311.1 \mathrm{~m} \mathrm{~T}$ $(g=2.150 \pm 0.005)$. This intense signal has been correlated with the $\mathrm{Cu}^{2+}$ paramagnetic center and attributed to a strong copper-hole delocalization on the four next-neighboring oxygen ions [28]. The g-factor of all of the samples was kept constant, but the $\Delta \mathrm{B}$ values (right inset in Fig. 3) increased and the signal intensities decreased as oxygen partial pressure decreased.

The decreases in the EPR signal indicate a decrease in the concentration of paramagnetic centers, while the broadening of lines may point to changes in type and/or concentration of different defects. Luo et al. suggest that this EPR signal in CCTO ceramics is directly correlated with copper-oxygen vacancy complex defect dipoles [29,30]. They have proposed that these complex defect dipoles are the main mechanism responsible for changes in intensity and $\Delta \mathrm{B}$ values in EPR signals in that they keep the g-factor constant in CCTO ceramics $[29,30]$. The following cation-oxygen vacancy complex defect dipoles are expected in the CCTO lattice due to the oxygen exchange:

$\left(\mathrm{Cu}_{\mathrm{Cu}}^{\prime}-\boldsymbol{V}_{\boldsymbol{O}}^{*}\right)^{\boldsymbol{x}}$

$\left(\mathrm{Cu}_{\mathrm{Cu}}^{\prime}-\mathrm{V}_{\boldsymbol{O}}-\mathrm{Cu}_{\mathrm{Cu}}^{\prime}\right)^{\boldsymbol{x}}$

Thus, the samples sintered at intermediate oxygen concentrations may have a high density of copper-oxygen vacancy complexes in the CCTO lattice, which leads to a lower EPR signal and broader EPR lines. The increase in oxygen concentration causes a decrease in the concentration of these complexes, thus resulting in a higher EPR signal and narrower EPR lines. In addition, the formation of surface $\mathrm{Ti}^{+3}$ cations has been reported in CCTO studies using x-ray photoelectron spectroscopy (XPS) [9,12], though EPR signals for $\mathrm{Ti}^{+3}$ paramagnetic centers with a gfactor usually lower than 2 were not observed [28,29]. Given the fact that EPR is a bulk technique, these results suggest that the concentration of $\mathrm{Ti}^{+3}$ cations and their related defects are below the detection limit for EPR.

Only the copper-related signal was identified using EPR in the CCTO phase, but several authors have reported that $\mathrm{T}_{\mathrm{u}}$ phonon modes, which are associated with the absorption regions of titanium and oxygen ions, are infrared active in the CCTO structure [31,32]. These active bands are associated with $v_{\mathrm{Ti}-\mathrm{O}}$ vibration at a range of $600-500 \mathrm{~cm}^{-1}$ and are associated with $\mathrm{v}_{\text {Ti-O-Ti }}$ stretching vibration at a range of $500-400 \mathrm{~cm}^{-1}$ [31,33]. The FTIR analyses of the CCTO samples presented in Fig. 4 

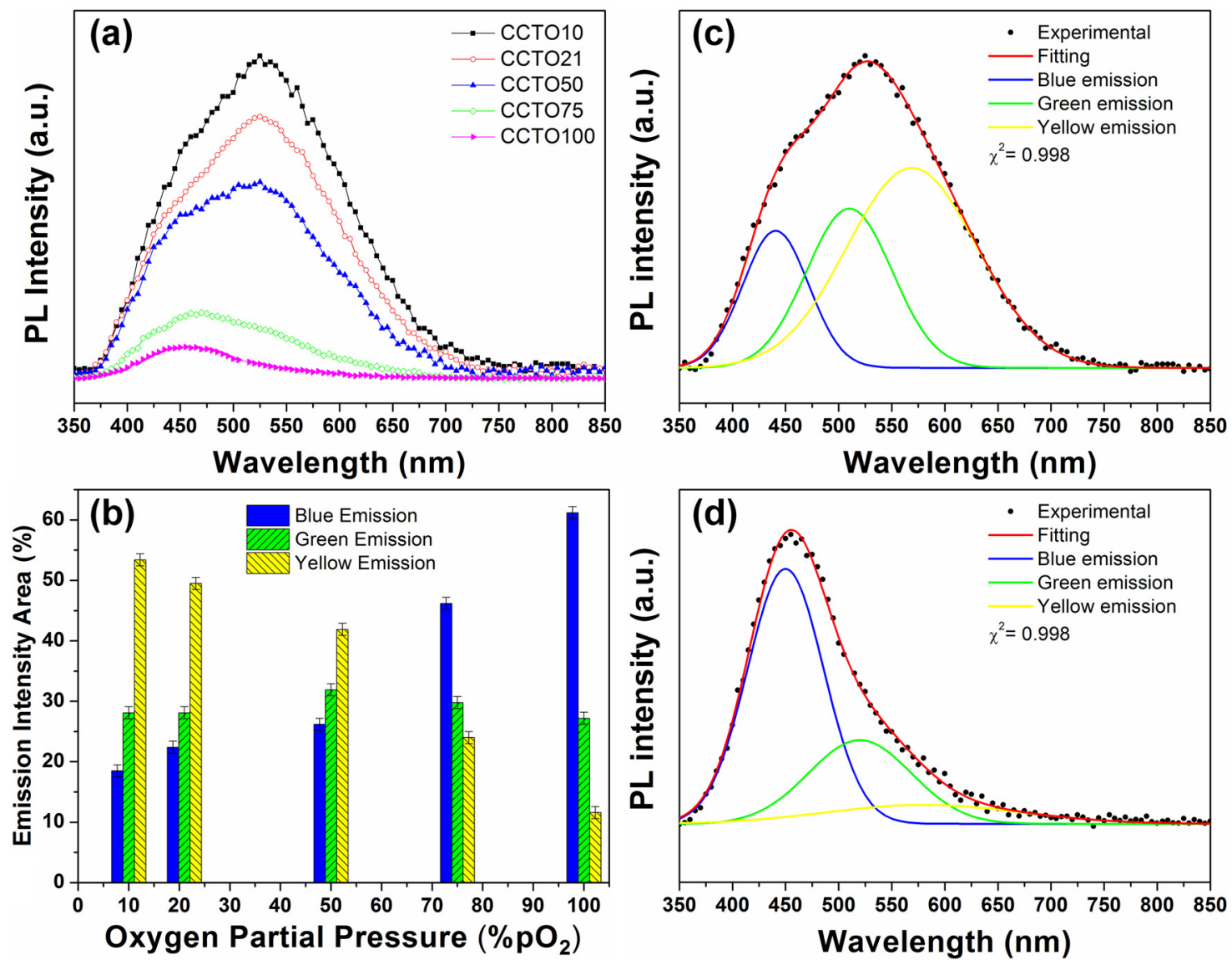

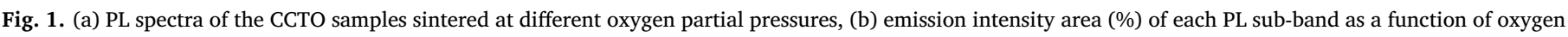
partial pressure, and the PL spectra deconvolution for (c) CCTO10 and (d) CCTO100.

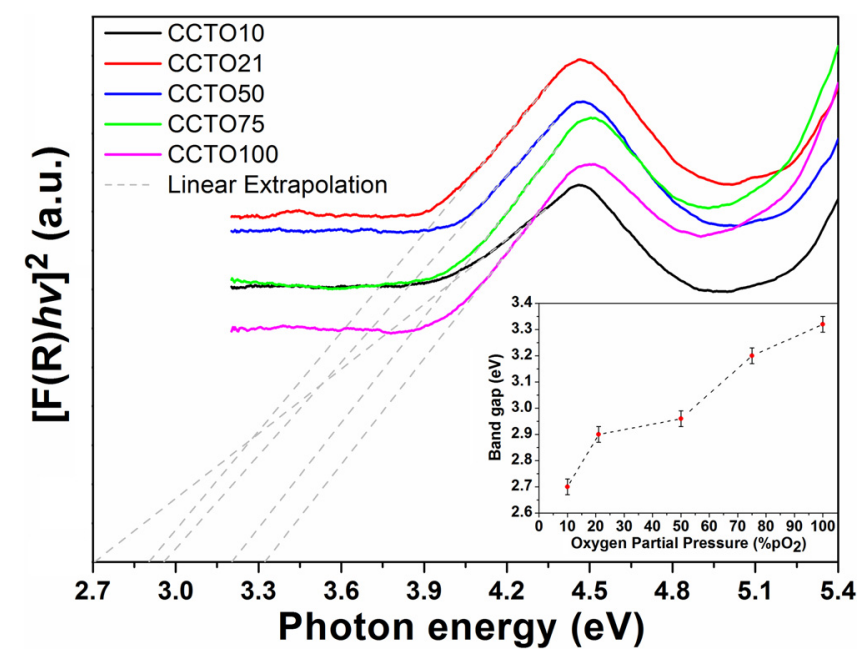

Fig. 2. UV-vis spectra of the CCTO samples sintered at different oxygen partial pressures. Right inset: Optical band gap values as a function of oxygen partial pressure.

revealed three major absorption bands centered around 542, 490, and $409 \mathrm{~cm}^{-1}$, but with a peak shift toward lower wavenumbers (see Figure S3f) when oxygen partial pressure decreased. As shown in Figure S3, the FTIR absorption spectra were fit using a pseudo-Voigt function only on the absorption peaks centered at 542 and $490 \mathrm{~cm}^{-1}$. Figs. $4 \mathrm{~b}$ and $4 \mathrm{c}$ show that the absorption intensity decreased as the oxygen partial pressure increased, a result which indicates a decrease in oxygen-titanium bonding, which itself may be reflective of the formation of oxygen

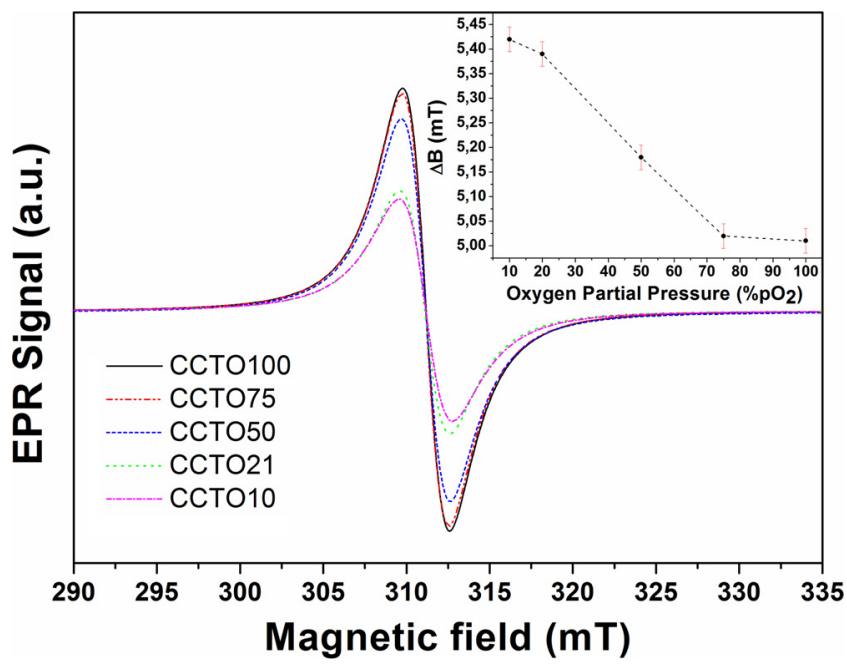

Fig. 3. EPR spectra of the CCTO samples sintered at different oxygen partial pressures. Right inset: $\Delta \mathrm{B}$ values as a function of oxygen partial pressure, extracted from the linewidth in the Lorentzian line-shape fitting (see Figure S2).

vacancies.

Recently, a theoretical model based on experimental evidence was proposed to explain PL emission in the $\mathrm{TiO}_{2}$ phase [34]. The formation of oxygen vacancies $\left(V_{o}^{x}, V_{o}^{\cdot}, V_{o}^{\cdot *}\right)$ was identified as the main mechanism responsible for the displacement of Ti-O chemical bonds and led to modifications in the bond lengths, bond angles and, consequently, distortions in the $\left[\mathrm{TiO}_{6}\right]$ octahedral clusters [34]. Thus, an increase in the concentration of oxygen vacancies in the CСTO lattice 

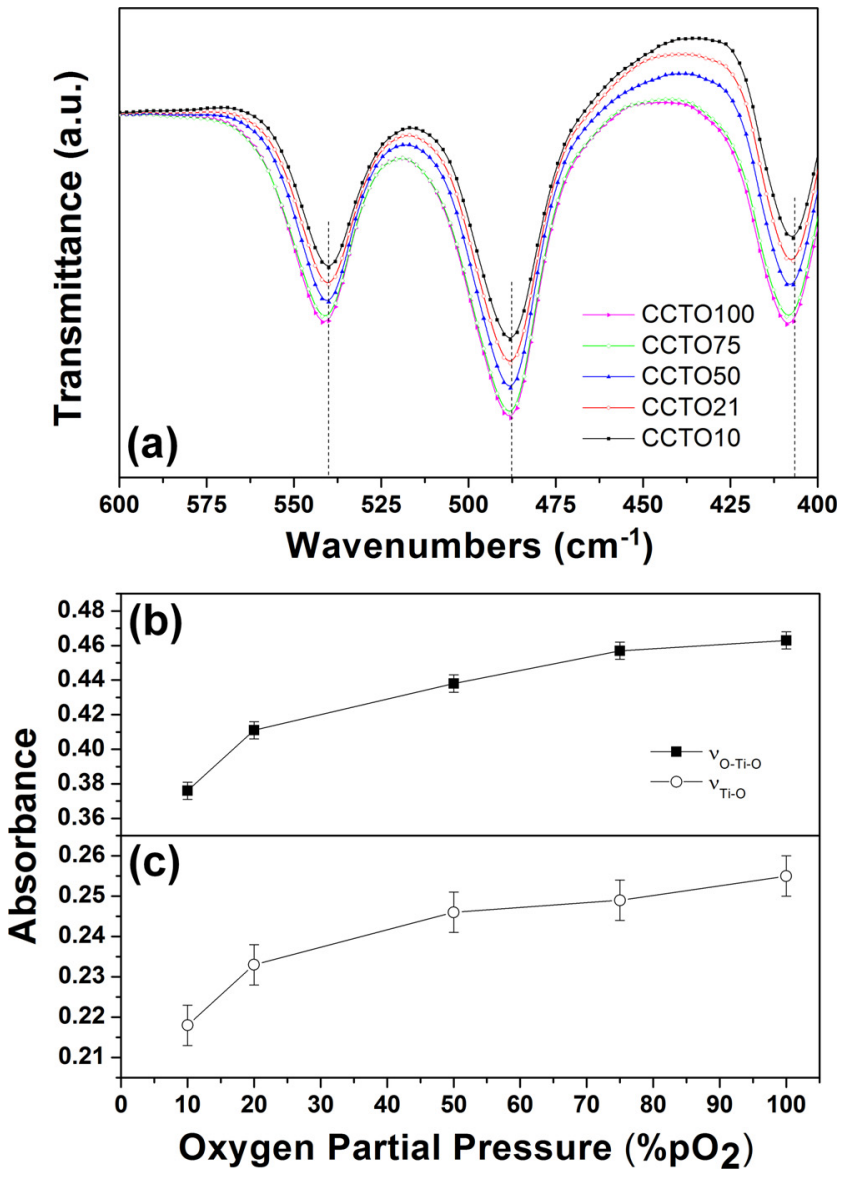

Fig. 4. (a) FTIR spectra of the CСTO samples sintered at different oxygen partial pressures. Absorbance intensity of the (b) $\mathrm{V}_{\mathrm{O}-\mathrm{Ti}}$ vibration $\left(542 \mathrm{~cm}^{-1}\right)$ and (c) $\mathrm{V}_{\text {Ti-O-Ti }}$ vibration $\left(490 \mathrm{~cm}^{-1}\right)$ as a function of oxygen partial pressure.

may result in a displacement of the Ti-O and O-Ti-O chemical bonds, thus leading to changes to the lattice parameter, bond lengths, and bond angles of the $\left[\mathrm{TiO}_{6}\right]$ octahedral clusters and to consequent shifts of the FTIR bands to low wavenumbers and decreases in absorption intensity [34,35]. In addition, the decrease of the copper EPR signal associated with the shifts of the FTIR bands to lower wavenumbers and decreases in absorption intensity all confirm the formation of oxygenrelated defects, which are concomitantly correlated with copper and titanium sites in the CСTO lattice. This finding also corroborates the formation of oxygen vacancies adjacent to both $\left[\mathrm{CuO}_{4}\right]$ and $\left[\mathrm{TiO}_{6}\right]$ clusters (and most probably $\left[\mathrm{CaO}_{12}\right]$ clusters) in the CCTO lattice, which are directly correlated with PL emission in this material [8].

PL emission in titanium oxide, the band emission of which is in the same visible electromagnetic range as CCTO, is composed of three subbands attributed to cation-oxygen clusters formed by the $\left[\mathrm{TiO}_{6}\right]$ octahedral clusters and different types of oxygen vacancies $\left(V_{o}^{x}, V_{o}^{\cdot}, V_{o}^{\cdot *}\right)$ [34]. Given the fact that the only parameter changing in our samples is the oxygen content during the sintering process, we can affirm that PL emission in the CСTO phase is also directly controlled by the formation of the different types of oxygen vacancies and that their relative concentrations depend on the amount of oxygen partial pressure during the sintering process. Thus, we can suggest that the high energy sub-band (blue emission), intermediate energy sub-band (green emission), and low energy sub-band (yellow emission) are related to and controlled by the respective formation of $V_{o}^{x}, V_{o}^{\cdot}$, and $V_{o}^{\cdot}$ oxygen vacancies in the CCTO phase $[8,22,23,34]$. The following mechanisms, in which $M_{M}^{x}$ is the cation located at a regular site in the crystalline lattice, are expected in the formation of oxygen-related defects in the ССТО lattice:
$\boldsymbol{O}_{O}^{x}+M_{M}^{x} \leftrightharpoons V_{o}^{\cdot}+M_{M}^{\prime}+\frac{1}{2} O_{2}(g)$

$O_{O}^{x}+2 M_{M}^{x} \leftrightharpoons V_{\ddot{O}}+2 M_{M}^{\prime}+\frac{1}{2} O_{2}(g)$

The compensation mechanisms for these equations have been proposed by many authors in the literature [14,15]. Indeed, the samples sintered above $50 \% \mathrm{pO}_{2}$ exhibited a high density of neutral oxygen vacancies $\left(V_{o}^{x}\right)$. This process leads to higher band gap values and lower overall PL intensity, the principal contribution of which involves the high energy sub-band (blue emission). Decreasing the oxygen concentration reduces band gap values, and PL intensity is dominated by the intermediate (green) sub-band and, in particular, by the low energy (yellow) subband, which are respectively associated with $V_{o}^{*}$ and $V_{o}^{*}$ oxygen vacancies. Taken together, the results for all of the samples tested show that the major PL sub-band energies are lower than their respective band gap values. This outcome indicates the presence of deep-level trap states below the conduction band. It can therefore also be suggested that band gap energy and PL emission are connected and are clearly associated with the formation of oxygen vacancies in the CCTO lattice; it is also likely that the deep-level trap states are modulated by the most types of oxygen vacancies.

In our previous study, the same samples were analyzed using XRPD and SEM. These experiments revealed that the CCTO phase is stable at oxygen-rich concentrations [17]. As the oxygen partial pressure decreases, the consequent loss of oxygen from the crystalline lattice results in non-stoichiometry of the CCTO matrix phase below $50 \% \mathrm{pO}_{2}$ due to the diffusion of copper to the grain boundary regions which, in turn, leads to a copper deficiency in the matrix phase. Meanwhile, at oxygen-poor concentrations, further reductions in the copper lead to complete decomposition of the ССТО phase. Thus, we believe that, at oxygen-rich concentrations, neutral oxygen vacancies are predominantly present and are associated with the formation of the stoichiometric CCTO phase. At intermediate oxygen concentrations, the monovalent and divalent oxygen vacancies shown in Eqs. (3) and (4) increased, and this increase is associated with ССТО non-stoichiometry. However, divalent oxygen vacancies are predominant in oxygen-intermediate concentrations, and the formation of these vacancies is also related to copper deficiency in the ССТО phase. This secondary mechanism may involve the formation of antisite defects in the CCTO lattice, as proposed by Sinclair et al. [14,15]. Our findings indicate that oxygen exchange and copper deficiency are the main mechanisms controlling the formation of oxygen-related defects and their concentrations in the ССТО phase, and that these mechanisms play the most important roles in the control and modulation of the optical and luminescence properties of polycrystalline CCTO ceramics.

\section{Conclusion}

Polycrystalline CCTO ceramics prepared by solid-state reaction at different oxygen partial pressures were investigated using bulk characterization techniques. The bulk spectroscopy, PL, UV-vis, FTIR, and EPR findings indicate the formation of defect complexes between acceptor centers with neutral, monovalent, and divalent oxygen vacancies due to oxygen exchange and copper deficiency in the CCTO lattice. This study confirmed that the formation of these oxygen-related defects and their concentrations are directly controlled by the oxygen partial pressure during the sintering process. In addition, these defects may modulate the optical and luminescence properties of polycrystalline ССТO ceramics. This behavior may be explained by the formation of different types of oxygen vacancies; it is possible that this process is the main mechanism responsible for the formation of deep-level trap states in the CCTO band gap. This research provides us with new insights into the role of oxygen in the formation of different types and concentrations of defects in the ССТО crystalline structure, as well as the influence of oxygen on the optical and luminescence properties of this phase. 
Subsequent studies are already underway to clarify the effect of these defects on the electrical and dielectric properties of CСTO material.

\section{Acknowledgements}

The authors would like to express their gratitude to Professor José A. Varela (in memoriam) for his invaluable contributions to the development of this research. The authors gratefully acknowledge the financial support from the São Paulo Research Foundation (FAPESP) (grant numbers 09/00367-6, 13/14647-6, and 13/07296-2) and the Brazilian National Council for Scientific and Technological Development (CNPq) (grant numbers: 2012/51195-3, 165902/2015-9, and $443138 / 2016-8$ ) in the development of this research. The authors would also like to thank C.F.O. Graeff and M.S. Li for their support with the EPR and PL characterization techniques, respectively.

\section{Appendix A. Supplementary data}

Supplementary material related to this article can be found, in the online version, at doi:https://doi.org/10.1016/j.jeurceramsoc.2018.06. 045 .

\section{References}

[1] A.P. Ramirez, M.A. Subramanian, M. Gardel, G. Blumberg, D. Li, T. Vogt, S.M. Shapiro, Giant dielectric constant response in a copper-titanate, Solid State Comm. 115 (5) (2000) 217-220.

[2] M.A. Subramanian, A.W. Sleight, ACu3Ti4O12 and ACu3Ru4O12 perovskites: high dielectric constants and valence degeneracy, Solid State Sci. 4 (3) (2002) 347-351.

[3] M.A. Subramanian, D. Li, N. Duan, B.A. Reisner, A.W. Sleight, High dielectric constant in ACu3Ti4O12 and ACu3Ti3FeO12 phases, J. Solid State Chem. 151 (2) (2000) 323-325.

[4] Y. Liu, R.L. Withers, X.Y. Wei, Structurally frustrated relaxor ferroelectric behavior in CaCu3Ti4O12, Phys. Rev. B 72 (13) (2005) 134104 4pp.

[5] D.C. Sinclair, T.B. Adams, F.D. Morrison, A.R. West, CaCu3Ti4012: one-step internal barrier layer capacitor, Appl. Phys. Lett. 80 (12) (2002) 2153-2155.

[6] T.T. Fang, H.K. Shiau, Mechanism for developing the boundary barrier layers of CaCu3Ti4O12, J. Am. Ceram. Soc. 87 (11) (2004) 2072-2079.

[7] P.R. Bueno, R. Tararam, R. Parra, E. Joanni, M.A. Ramirez, W.C. Ribeiro, E. Longo, J.A. Varela, A polaronic stacking fault defect model for CaCu3Ti4O12 material: an approach for the origin of the huge dielectric constant and semiconducting coexistent features, J. Phys. D: Appl. Phys. 42 (5) (2009) 055404 9pp.

[8] L.H. Oliveira, E.C. Paris, W. Avansi, M.A. Ramirez, V.R. Mastelaro, E. Longo, J.A. Varela, Correlation between photoluminescence and structural defects in Ca1xCu3-xTi4O12 systems, J. Am. Ceram. Soc. 96 (1) (2013) 209-217.

[9] A.A. Felix, E. Longo, J.A. Varela, M.O. Orlandi, Gas sensing and conductivity relationship on nanoporous thin films: a CaCu3Ti4012 case study, Thin Solid Films 604 (19) (2016) 69-73.

[10] L. Zhang, Z.L. Tang, Polaron relaxation and variable-range-hopping conductivity in the giant-dielectric-constant material CaCu3Ti4012, Phys. Rev. B 70 (17) (2004) 174306 6pp.

[11] R.A. Eichel, Structural and dynamic properties of oxygen vacancies in perovskite oxides-analysis of defect chemistry by modern multi-frequency and pulsed EPR techniques, Phys. Chem. Chem. Phys. 13 (2) (2011) 368-384.

[12] G. Deng, N. Xanthopoulos, P. Muralt, Chemical nature of colossal dielectric constant of CaCu3Ti4O12 thin film by pulsed laser deposition, Appl. Phys. Lett. 92 (17) (2008) 172909 3pp.
[13] T.T. Fang, L.T. Mei, H.F. Ho, Effects of Cu stoichiometry on the microstructures, barrier-layer structures, electrical conduction, dielectric responses, and stability of CaCu3Ti4O12, Acta Mater. 54 (10) (2006) 2867-2875.

[14] M.J. Abu, J.J. Mohamed, M.F. Ain, Z.A. Ahmad, Phase structure, microstructure and broadband dielectric response of $\mathrm{Cu}$ nonstoichiometry CaCu3Ti4O12 ceramic, J. Alloys Compd. 683 (2016) 579-589.

[15] R. Schmidt, S. Pandey, P. Fiorenza, D.C. Sinclair, Non-stoichiometry in CaCu3Ti4O12 (CCTO) ceramic, RSC Adv. 3 (34) (2013) 14580-14589.

[16] L.H. Oliveira, A.P. de Moura, F.A. La Porta, I.C. Nogueira, E.C. Aguiar, T. Sequinel, I.L.V. Rosa, E. Longo, J.A. Varela, Influence of Cu-doping on the structural and optical properties of CaTiO3 powders, Mater. Res. Bull. 81 (2016) 1-9.

[17] A.A. Felix, V.D.N. Bezzon, M.O. Orlandi, D. Vengust, M. Spreitzer, E. Longo, D. Suvorov, J.A. Varela, Role of oxygen on the phase stability and microstructure evolution of CaCu3Ti4O12 ceramics, J. Eur. Ceram. Soc. 37 (1) (2017) 129-136.

[18] A.A. Felix, M.O. Orlandi, J.A. Varela, Schottky-type grain boundaries in CCTO ceramics, Solid State Commun. 151 (19) (2011) 1377-1381.

[19] G. Blasse, B.C. Grabmaier, Luminescent Materials, 1st ed., Springer, Berlin, 1994.

[20] I.C. Nogueira, L.S. Cavalcante, P.F.S. Pereira, M.M. de Jesus, J.M.R. Mercury, N.C. Batista, M.S. Li, E. Longo, Rietveld refinement, morphology and optical properties of (Ba1-xSrx)MoO4 crystals, J. Appl. Cryst. 46 (2013) 1434-1446.

[21] Y.Y. Tay, T.T. Tan, F. Boey, M.H. Liang, J. Ye, Y. Zhao, T. Norby, S. Li, Correlation between the characteristic green emissions and specific defects of $\mathrm{ZnO}$, Phys. Chem. Chem. Phys. 12 (10) (2010) 2373-2379.

[22] S. Lany, A. Zunger, Dopability, intrinsic conductivity, and nonstoichiometry of transparent conducting oxides, Phys. Rev. Lett. 98 (4) (2007) 045501 4pp.

[23] S. Lazaro, J. Milanez, A.T. Figueiredo, V. Longo, V.R. Mastelaro, F.S. Vicente, A.C. Hernandes, J.A. Varela, E. Longo, Relation between photoluminescence emission and local order-disorder in the CaTiO3 lattice modifier, Appl. Phys. Lett. 90 (11) (2007) 111904 3pp.

[24] H.B. Xiao, C.P. Yang, C. Huang, L.F. Xu, D.W. Shi, V.V. Marchenkov, I.V. Medvedeva, K. Bärner, Influence of oxygen vacancy on the electronic structure of CaCu3Ti4O12 and its deep-level vacancy trap states by first-principle calculation, J. Appl. Phys. 111 (6) (2012) 063713 8pp.

[25] R.A. Smith, Semiconductors, 2nd ed., Cambridge University Press, London, 1978.

[26] B. Henderson, G.F. Imbusch, Optical Spectroscopy of Inorganic Solids, 1st ed., University Press, Oxford, Oxford, 1989.

[27] R.A. Eichel, Characterization of defect structure in acceptor-modified piezoelectric ceramics by multifrequency and multipulse electron paramagnetic resonance spectroscopy, J. Am. Ceram. Soc. 91 (3) (2008) 691-701.

[28] M.C. Mozzati, C.B. Azzoni, D. Capsoni, M. Bini, V. Massarotti, Electron paramagnetic resonance investigation of polycrystalline CaCu3Ti4012, J. Phys. Condens. Matter 15 (43) (2003) 7365-7374.

[29] X.J. Luo, K. Barner, S.L. Tang, C.P. Yang, Y.W. Du, Electron paramagnetic resonance probed defects in the colossal dielectric constant perovskite CaCu3Ti4O12, J. Phys. Soc. Jpn. 82 (6) (2013) 064707 5pp.

[30] X.J. Luo, Y.S. Liu, C.P. Yang, S.S. Chen, S.L. Tang, K. Barner, Oxygen vacancy related defect dipoles in CaCu3Ti4O12: detected by electron paramagnetic resonance spectroscopy, J. Eur. Ceram. Soc. 35 (7) (2015) 2073-2081.

[31] L.X. He, J.B. Neaton, M.H. Cohen, D. Vanderbilt, C.C. Homes, First-principles study of the structure and lattice dielectric response of CaCu3Ti4O12, Phys. Rev. B 65 (21) (2002) 214112 11pp.

[32] N. Kolev, R.P. Bontchev, A.J. Jacobson, V.N. Popov, V.G. Hadjiev, A.P. Litvinchuk, M.N. Iliev, Raman spectroscopy of CaCu3Ti4O12, Phys. Rev. B 66 (13) (2002) $1321024 \mathrm{pp}$.

[33] L.C. Kretly, A.F.L. Almeida, P.B.A. Fechine, R.S. de Oliveira, A.S.B. Sombra, Dielectric permittivity and loss of CaCu3Ti4O12 (CCTO) substrates for microwave devices and antennas, J. Mater. Sci.: Mater. Electron. 15 (10) (2004) 657-663.

[34] E. Silva Junior, F.A. La Porta, M.S. Li, J. Andrés, J.A. Varela, E. Longo, A relationship between structural and electronic order-disorder effects and optical properties in crystalline TiO2 nanomaterials, Dalton Trans. 44 (7) (2015) 3159-3175.

[35] J. Li, M.A. Subramanian, H.D. Rosenfeld, C.Y. Jones, B.H. Toby, A.W. Sleight, Clues to the giant dielectric Constant of CaCu3Ti4O12 in the defect structure of SrCu3Ti4O12, Chem. Mater. 16 (25) (2004) 5223-5225. 